\title{
On Quasi-equally Spaced Sampling in Wireless Sensor Networks
}

(Invited paper)

\author{
Alessandro Nordio \\ Politecnico di Torino \\ Torino, Italy \\ Email: alessandro.nordiopolito.it
}

\author{
Carla Chiasserini \\ Politecnico di Torino \\ Torino, Italy \\ Email: carla.chiasserinipolito.it
}

\author{
Emanuele Viterbo \\ DEIS, University of Calabria \\ Arcavacata di Rende, Italy \\ Email: viterbodeis.unical.it
}

\begin{abstract}
In this paper we study wireless sensor networks for monitoring applications. We focus on the problem of sampling and reconstruction of multidimensional bandlimited signals, when the sensor locations are equally spaced points affected by some jitter, and the sensor measurements are affected by noise. We show how the mean square reconstruction error can be estimated from the eigenvalue distribution of a certain Toeplitz matrix. We analyze the $d$-dimensional case, and we show how the mean square error can be easily estimated by using asymptotic analysis.
\end{abstract}

\section{INTRODUCTION}

Sensor networks, whose nodes sample a physical field, like air temperature, light intensity, pollution levels or rain falls, typically represent an example of quasi-equally spaced sampling [1]-[4]. Indeed, in general, sensors are not regularly deployed in the area of interest due to terrain conditions and deployment practicality and, thus, the physical field is not regularly sampled in the space domain.

We consider that sensors report their measurements to a common processing unit (or sink node), which is in charge of reconstructing the sensed field, based on the received samples and on the knowledge of their coordinates. If the field is bandlimited in the space domain, then an estimate of the discrete spectrum can be obtained by using linear reconstruction techniques [1], [5], even in presence of additive noise. We assume a $d$-dimensional physical field $(d \geq 1)$, which can be represented with a certain number of harmonics per dimension.

A convenient parameter commonly used to measure the performance of such technique is the mean square error (MSE) on the $d$-dimensional reconstructed signal. In [1], [6] it has been shown that the asymptotic MSE (i.e., as the number of harmonics and the number of samples tend to infinity while their ratio is kept constant) is a function of the asymptotic eigenvalue distribution $f_{\lambda}$ of a particular Toeplitz matrix $\mathbf{T}_{d}$, which is related to the sampling system.

While several results have appeared in the literature for Gaussian matrices or matrices with independent entries [7], [8], few results are known for the matrices like the ones studied in this paper. In particular, the asymptotic eigenvalue distribution of $\mathbf{T}_{d}$ is still unknown. Here, we derive the moments of $f_{\lambda}$, which are a useful basis for approximating the distribution and for performing deconvolution [9], [10]. Furthermore, we show that, as $d \rightarrow \infty, f_{\lambda}$ tends to the Marčenko-Pastur distribution [11]. Finally, we present some numerical results and applications where our findings are of great use.

\section{SySTEM MODEL AND PREVIOUS RESULTS}

As a first step, let us consider a one-dimensional field which, when observed over a finite interval, it admits an infinite Fourier series expansion [6]. However, one can think of the largest index $M$ of the non-negligible Fourier coefficients of the expansion as the approximate one-sided bandwidth of the field. We therefore represent the one-dimensional field, $s(x)$, by using $2 M+1$ harmonics as

$$
s(x)=\frac{1}{\sqrt{2 M+1}} \sum_{k=-M}^{M} a_{k} \mathrm{e}^{\mathrm{j} 2 \pi k x}
$$

The field is observed within one period interval $[0,1)$ and sampled by $r$ sensors placed at positions ${ }^{1} \mathbf{x}=\left[x_{0}, \ldots, x_{r-1}\right]^{\top}$, $x_{q} \in[0,1), q=0, \ldots, r-1$, which are independent random variables with distribution $f_{x_{q}}(z), 0 \leq z<1$. The signal samples are denoted by the column vector $\mathbf{s}=$ $\left[s\left(x_{0}\right), \ldots, s\left(x_{r-1}\right)\right]^{\top}$. The field discrete spectrum is given by the $2 M+1$ complex vector $\mathbf{a}=\left[a_{-M}, \ldots, a_{0}, \ldots, a_{M}\right]^{T}$. The complex numbers $a_{k}$ represent amplitudes and phases of the harmonics in $s(x)$.

The vector $\mathbf{s}$ is related to the spectrum a through the expression

$$
\mathbf{s}=\mathbf{G}_{\mathbf{x}}^{\dagger} \mathbf{a}
$$

where the matrix $\mathbf{G}$ has entries

$$
(\mathbf{G})_{k q}=\frac{1}{\sqrt{2 M+1}} \mathrm{e}^{-\mathrm{j} 2 \pi k x_{q}} \quad \begin{aligned}
k & =-M, \ldots, M \\
q & =0, \ldots, r-1
\end{aligned}
$$

\footnotetext{
${ }^{1}$ Column vectors and matrices are denoted by bold lowercase and bold upper case letters, respectively. $(\mathbf{X})_{k q}$ is the $(k, q)$ entry of the matrix $\mathbf{X}$, and $\mathbf{I}$ is the generic identity matrix. The conjugate transpose operator is denoted by $(\cdot)^{\dagger}$
} 
Note that these matrices appear in many signal processing applications and they have been studied in a number of recent works, e.g., [1], [6], [12]-[15]. More specifically, the work in [1] considered the case where sampling positions are subject to an unknown jitter, while [15] presented several applications of the above Vandermonde matrix, which range from multiuser MIMO systems to multifold scattering.

Now, consider linear techniques for reconstructing a signal from the set of $r$ irregularly-spaced samples with known coordinates. In [6], [13]-[15], it has been observed that the performance of these techniques are functions of the asymptotic eigenvalue distribution of the Hermitian Toeplitz matrix $\mathbf{T}=\beta \mathbf{G G}^{\dagger}$, where

$$
\beta=\frac{2 M+1}{r}
$$

is the aspect ratio (i.e., the ratio between the number of rows and the number of columns) of the matrix $\mathbf{G}$. The asymptotic eigenvalue distribution of $\mathbf{T}$ is defined as the distribution of its eigenvalues, $f_{\lambda}(\beta, z)$, in the limit of $M$ and $r$ growing to infinity with constant aspect ratio $\beta$.

Unfortunately, such eigenvalue distribution is still unknown. However, an explicit expression of the moments

$$
\mathbb{E}\left[\lambda^{p}\right]=\int_{0}^{\infty} z^{p} f_{\lambda}(\beta, z) \mathrm{d} z
$$

has been first derived in [12], when $x_{q}$ are uniformly distributed in $[0,1)$. Also, in the case where $x_{q}$ are independent, quasi-equally spaced random variables, the analytic expression of the second moment of the eigenvalue distribution of $\mathbf{T}$, i.e., $\mathbb{E}\left[\lambda^{2}\right]$, has been obtained in [1]. Then, in [14] the moments $f_{\lambda}(\beta, z)$ have been derived for the one-dimensional case and any distribution $f_{x_{q}}(z)$, such that $f_{x_{q}}(z)=f_{x}(z)$, $q=0, \ldots, r-1$.

In this work, we push the analysis of the matrix $\mathbf{G}$ further and consider a more general formulation, which extends the model in (1) to the multidimensional domain. We therefore study the properties of $(2 M+1)^{d} \times r$ random matrices with entries given by

$$
\left(\mathbf{G}_{d}\right)_{\nu(\ell), q}=\frac{1}{\sqrt{(2 M+1)^{d}}} \mathrm{e}^{-\mathrm{j} 2 \pi \ell^{\top} \mathbf{x}_{q}}
$$

where the vectors $\mathbf{x}_{q}=\left[x_{q 1}, \ldots, x_{q d}\right]^{\top}$ have independent entries, characterized by the pdf $f_{x_{q m}}(z), q=0, \ldots, r-1$, $m=1, \ldots, d$. The invertible function

$$
\nu(\ell)=\sum_{m=1}^{d}(2 M+1)^{m-1} \ell_{m}
$$

maps the vector of integers $\ell=\left[\ell_{1}, \ldots, \ell_{d}\right]^{\top}, \ell_{m}=$ $-M, \ldots, M$ onto a scalar index, i.e., the row index of the matrix $\mathbf{G}_{d}$. In [12] it has been shown that the performance of linear reconstruction techniques in sensor networks still depends on the asymptotic eigenvalue distribution $f_{\lambda}(d, \beta, z)$ of the matrix $\mathbf{T}_{d}=\beta \mathbf{G}_{d} \mathbf{G}_{d}^{\dagger}$. Notice that in the $d$-dimensional case, $\beta=\frac{(2 M+1)^{d}}{r}$ For given $\beta$ and $d$, the analytic expression of $f_{\lambda}(d, \beta, z)$ is still unknown, however, under the assumption of $\mathbf{x}_{q}$ uniformly distributed over $[0,1)^{d}$, we derived the analytic expression of its moments [12]. Under the same assumptions, in [12] we showed that as $d \rightarrow \infty, f_{\lambda}(d, \beta, z)$ tends to the Marčenko-Pastur law [11], i.e.,

$$
\lim _{d \rightarrow \infty} f_{\lambda}(d, \beta, z)=f_{\infty}(\beta, z)=\frac{\sqrt{\left(c_{1}-z\right)\left(z-c_{2}\right)}}{2 \pi z \beta}
$$

where $c_{1}, c_{2}=(1 \pm \sqrt{\beta})^{2}, 0<\beta \leq 1, c_{2} \leq z \leq c_{1}$

Next, we detail the problem addressed in this work and introduce some useful notations.

\section{Problem formulation: THE QUASI-EQUally SPACED MULTIDIMENSIONAL MODEL}

Let us consider that the vectors $\mathrm{x}$ are independent, quasiequally spaced random variables in the $d$-dimensional hypercube $[0,1)^{d}$, i.e., the averages of $\mathbf{x}$ represent the coordinate of the vertices of a $d$-dimensional grid in $[0,1)^{d}$. This is often the case in measurement systems affected by jitter, or in sensor network deployments, where the devices sampling the field of interest cannot be regularly positioned due to terrain conditions and deployment practicality [2]. Note that the distribution of the variables $\mathrm{x}$ can be of any kind, the only assumption we make is on their averages being equally spaced. Again, under these conditions, the performance of a sampling system can be expressed by using the eigenvalue distribution of the matrix $\mathbf{T}_{d}=\beta \mathbf{G}_{d} \mathbf{G}_{d}^{\dagger}$, i.e., $f_{\lambda}(d, \beta, z)[1]$.

We define $\rho$ as the number of samples per dimension, thus the total number of samples is $r=\rho^{d}$. We denote the coordinate of a generic vertex of the grid by the vector $\mathbf{q} / \rho \in[0,1)^{d}$, where $\mathbf{q}=\left[q_{1}, \ldots, q_{d}\right]^{\top}, q_{m}=0, \ldots, \rho-1$. For notation simplicity and in analogy with (5), we identify the vertex with coordinate $\mathbf{q} / \rho$ by the scalar index

$$
\mu(\mathbf{q})=\sum_{m=1}^{d} \rho^{m-1} q_{m}
$$

Notice that $0 \leq \mu(\mathbf{q}) \leq r-1$ is an invertible function and allows us to write

$$
\mathbf{x}_{\mu(\mathbf{q})}=\frac{\mathbf{q}}{\rho}+\frac{\tilde{\mathbf{x}}_{\mu(\mathbf{q})}}{\rho}
$$

where

$$
\mathbb{E}\left[\mathbf{x}_{\mu(\mathbf{q})}\right]=\frac{\mathbf{q}}{\rho}
$$

is the average coordinate of the sample identified by the scalar label $\mu(\mathbf{q})$. Furthermore, we assume that the entries of the vectors $\tilde{\mathbf{x}}_{\mu(\mathbf{q})}$ are i.i.d with pdf $f_{\tilde{x}}(z)$ and do not depend on $d, r, M$, or $\mathbf{q}$. By using this notation, the entries of $\mathbf{G}_{d}$ are then given by

$$
\left(\mathbf{G}_{d}\right)_{\nu(\ell), \mu(\mathbf{q})}=\frac{1}{\sqrt{(2 M+1)^{d}}} \mathrm{e}^{-\mathrm{j} 2 \pi \ell^{\top} \mathbf{x}_{\mu(\mathbf{q})}}
$$

while the aspect ratio is

$$
\beta=\frac{(2 M+1)^{d}}{r}=\left(\frac{2 M+1}{\rho}\right)^{d}
$$


The Hermitian Toeplitz matrix $\mathbf{T}_{d}=\beta \mathbf{G}_{d} \mathbf{G}_{d}^{\dagger}$ is defined as

$$
\left(\mathbf{T}_{d}\right)_{\nu(\ell), \nu\left(\ell^{\prime}\right)}=\frac{1}{\rho^{d}} \sum_{\mathbf{q}} \mathrm{e}^{-\mathrm{j} 2 \pi \mathbf{x}_{\mu(\mathbf{q})}^{\top}\left(\boldsymbol{\ell}-\boldsymbol{\ell}^{\prime}\right)}
$$

where $\sum_{\mathbf{q}}$ is a $d$-dimensional sum over all vectors $\mathbf{q}$ such that $q_{m}=0, \ldots, \rho-1, m=1, \ldots, d$.

In the following section, we derive the analytic expression of the moments of $f_{\lambda}(d, \beta, z)$ with quasi-equally spaced vectors $\mathbf{x}_{\mu}(\mathbf{q})$. Then, in Section $\mathrm{V}$ we show that as $d \rightarrow \infty, f_{\lambda}(d, \beta, z)$ tends to the Marčenko-Pastur law.

\section{Closed Form EXPRESSION OF THE MOMENTS OF THE ASYMPTOTIC EIGENVALUE PDF}

Following the approach adopted in [16], [17], in the limit for $M$ and $r$ growing to infinity with constant aspect ratio $\beta$ and dimension $d$, we compute the closed form expression of $\mathbb{E}\left[\lambda^{p}\right]$, which can be obtained from the powers of $\mathbf{T}_{d}$ as [7],

$$
\mathbb{E}\left[\lambda^{p}\right]=\lim _{M, r \rightarrow+\infty} \frac{\operatorname{Tr}\left\{\underset{\mathcal{X}}{\mathbb{E}}\left[\mathbf{T}_{d}^{p}\right]\right\}}{(2 M+1)^{d}}
$$

where the symbol Tr identifies the matrix trace operator, and the average $\underset{\mathcal{X}}{\mathbb{E}}[\cdot]$ is computed over the set of random variables $\mathcal{X}=\left\{\mathbf{x}_{0}, \ldots, \mathbf{x}_{r-1}\right\}$. An efficient method to compute (10) exploits set partitioning. Indeed, the power $\mathbf{T}_{d}^{p}$ is the matrix product of $p$ copies of $\mathbf{T}_{d}$. This operation yields exponential terms, whose exponents are given by a combination of $p$ terms of the form $\mathbf{x}_{\mu\left(\mathbf{q}_{i}\right)}^{\mathrm{T}}\left(\boldsymbol{\ell}_{i}-\boldsymbol{\ell}_{[i+1]}\right)$ (the proof is omitted for space limitations). Each of these combinations can be represented as a set of elements from the set $\mathcal{P}=\{1, \ldots, p\}$. In particular, a combination, where $k$ terms out of $p$ take distinct values, corresponds to a subset of $\mathcal{P}$ of size $k$. Therefore, a fundamental step to calculate (10) is the computation of all possible partitions of set $\mathcal{P}$. To this goal, before proceeding further in our analysis, we introduce some useful definitions related to set partitioning.

\section{A. Definitions}

Let the integer $p$ be the moment order and vector $\boldsymbol{\mu}$ be a possible combination of $p$ integers. We define

- the scalar integer $1 \leq k(\boldsymbol{\mu}) \leq p$ as the number of entries of the vector $\boldsymbol{\mu}$ taking distinct values;

- $\boldsymbol{\gamma}(\boldsymbol{\mu})$ as the vector of integers, of length $k(\boldsymbol{\mu})$, whose entries $\gamma_{j}(\boldsymbol{\mu}), j=1, \ldots, k(\boldsymbol{\mu})$, are the entries of $\boldsymbol{\mu}$ without repetitions, in order of appearance within $\boldsymbol{\mu}$;

- $\mathcal{P}_{j}(\boldsymbol{\mu})$ as the set of indices of the entries of $\boldsymbol{\mu}$ with value $\gamma_{j}(\boldsymbol{\mu}), j=1, \ldots, k(\boldsymbol{\mu})$;

- the vector $\boldsymbol{\omega}(\boldsymbol{\mu})=\left[\omega_{1}(\boldsymbol{\mu}), \ldots, \omega_{p}(\boldsymbol{\mu})\right]$ where $\omega_{i}(\boldsymbol{\mu})=j$ if $i \in \mathcal{P}_{j}(\boldsymbol{\mu}), i=1, \ldots, p, j=1, \ldots, k(\boldsymbol{\mu})$.
Example 1: Let $\boldsymbol{\mu}=[1,5,2,8,5,3,2]$, then $k(\boldsymbol{\mu})=5$ since the entries of $\boldsymbol{\mu}$ take 5 distinct values (i.e., $\{1,5,2,8,3\}), \gamma(\boldsymbol{\mu})=[1,5,2,8,3]$, and $\mathcal{P}_{1}(\boldsymbol{\mu})=\{1\}$, $\mathcal{P}_{2}(\boldsymbol{\mu})=\{2,5\}, \mathcal{P}_{3}(\boldsymbol{\mu})=\{3,7\}, \mathcal{P}_{4}(\boldsymbol{\mu})=\{4\}$, $\mathcal{P}_{5}(\boldsymbol{\mu})=\{6\}$. The partition identified by $\boldsymbol{\mu}$ is therefore $\{\{1\},\{2,5\},\{3,7\},\{4\},\{6\}\}$ or, equivalently, it is given by $\boldsymbol{\omega}(\boldsymbol{\mu})=[1,2,3,4,2,5,3]$.

Also, we define:

- $\Omega_{p}$ as the set of partitions of the $\mathcal{P}$;

- $\Omega_{p, k}$ as the set of partitions of $\mathcal{P}$ in $k$ subsets, $1 \leq k \leq p$, with $\bigcup_{k=1}^{p} \Omega_{p, k}=\Omega_{p}$.

Note that: $(i)$ the cardinality of $\Omega_{p}$, denoted by $B(p)=\left|\Omega_{p}\right|$, is the $p$-th Bell number [18] and (ii) the cardinality of $\Omega_{p, k}$, denoted by $S(p, k)=\left|\Omega_{p, k}\right|$, is a Stirling number of the second kind [19].

Example 2: Let $p=3$. Then, the set $\Omega_{3}$ has cardinality $B(3)=5$ and the sets $\Omega_{3,1}, \Omega_{3,2}, \Omega_{3,3}$ have cardinality $S(3,1)=1, S(3,2)=3$ and $S(3,3)=$ 1 , respectively. In particular, $\Omega_{3,1}=\{\{1,2,3\}\}$, $\Omega_{3,2}=\{\{\{1,2\},\{3\}\},\{\{1,3\},\{2\}\},\{\{1\},\{2,3\}\}\}$, and $\Omega_{3,3}=\{\{1\},\{2\},\{3\}\}$.

From the above definitions, it follows that:

1) the vector $\boldsymbol{\mu}$ induces a partition of the set $\mathcal{P}$ which is identified by the subsets $\mathcal{P}_{j}(\boldsymbol{\mu})$. These subsets have the following properties

$$
\bigcup_{j=1}^{k(\boldsymbol{\mu})} \mathcal{P}_{j}(\boldsymbol{\mu})=\mathcal{P}, \quad \mathcal{P}_{j}(\boldsymbol{\mu}) \cap \mathcal{P}_{j^{\prime}}(\boldsymbol{\mu})=\emptyset
$$

for $j \neq j^{\prime}$. Even though the partition identified by $\boldsymbol{\mu}$ is often represented as $\left\{\mathcal{P}_{1}, \ldots, \mathcal{P}_{k(\boldsymbol{\mu})}\right\}$, by its definition, an equivalent representation of such partition is given by the vector $\boldsymbol{\omega}(\boldsymbol{\mu})$. Therefore, from now on we will refer to $\boldsymbol{\omega}(\boldsymbol{\mu})$ as a partition of the $p$ element set $\mathcal{P}$ induced by $\boldsymbol{\mu}$ (for simplicity, however, we will not explicit the dependency on $\boldsymbol{\mu}$ );

2) $k(\boldsymbol{\omega})=k(\boldsymbol{\mu})$, since the entries of $\boldsymbol{\omega}$ take all possible values in the set $\{1, \ldots, k(\boldsymbol{\mu})\}$;

3) $\mathcal{P}_{j}(\boldsymbol{\omega})=\mathcal{P}_{j}(\boldsymbol{\mu})$, for $j=1, \ldots, k(\boldsymbol{\mu})$.

\section{B. Closed form expression of $\mathbb{E}\left[\lambda^{p}\right]$}

By using the definitions in Section IV-A and by applying set partitioning to (10), we can state the first main result of this work:

Theorem 4.1: Let $\mathbf{T}_{d}$ be a $(2 M+1)^{d} \times(2 M+1)^{d}$ Hermitian random matrix as defined in (9), where the properties of the random vectors $\mathbf{x}_{\mu(\mathbf{q})}$ are described in Section III. Then, for any given $\beta$ and $d$, the $p$-th moment of the asymptotic eigenvalue distribution of $\mathbf{T}_{d}$ is given by:

$$
\mathbb{E}\left[\lambda^{p}\right]=\sum_{k=1}^{p} \sum_{h=1}^{k} \beta^{p-h} \sum_{\boldsymbol{\omega} \in \Omega_{p, k}} \sum_{\boldsymbol{\omega}^{\prime} \in \Omega_{k, h}} u\left(\boldsymbol{\omega}^{\prime}\right) v\left(\boldsymbol{\omega}, \boldsymbol{\omega}^{\prime}\right)^{d}
$$


where

$$
\begin{aligned}
& u\left(\boldsymbol{\omega}^{\prime}\right)=(-1)^{k-h} \prod_{j^{\prime}=1}^{h}\left(\left|\mathcal{P}_{j^{\prime}}\left(\boldsymbol{\omega}^{\prime}\right)\right|-1\right) ! \\
& v\left(\boldsymbol{\omega}, \boldsymbol{\omega}^{\prime}\right)= \begin{cases}\int_{\mathcal{H}_{p}} \prod_{j=1}^{k} C\left(-\mathrm{j} 2 \pi \beta^{1 / d} w_{j}(\boldsymbol{\omega})\right) \mathrm{d} \mathbf{y} & h=1 \\
\int_{\mathcal{H}_{p}} \prod_{j=1}^{k} C\left(-\mathrm{j} 2 \pi \beta^{1 / d} w_{j}(\boldsymbol{\omega})\right) & \\
\times \prod_{j^{\prime}=1}^{h} \delta_{D}\left(\sum_{i^{\prime} \in \mathcal{P}_{j^{\prime}}\left(\boldsymbol{\omega}^{\prime}\right)} w_{i^{\prime}}(\boldsymbol{\omega})\right) \mathrm{d} \mathbf{y} & 1<h<k \\
\int_{\mathcal{H}_{p}} \prod_{j=1}^{k} \delta_{D}\left(w_{j}(\boldsymbol{\omega})\right) \mathrm{d} \mathbf{y} & \\
& h=k\end{cases}
\end{aligned}
$$

and $v\left(\boldsymbol{\omega}, \boldsymbol{\omega}^{\prime}\right)=1$ for $k=1$. We defined $\mathcal{H}_{p}$ as the $p$ dimensional hypercube $[-1 / 2,1 / 2)^{p}, C(s)=\underset{\tilde{x}}{\mathbb{E}}\left[\mathrm{e}^{s z}\right]$ as the characteristic function of $\tilde{x}, \delta_{D}(\cdot)$ as the Dirac's delta, and

$$
w_{j}(\boldsymbol{\omega})=\sum_{i \in \mathcal{P}_{j}(\boldsymbol{\omega})} y_{i}-y_{[i+1]}
$$

$y_{i} \in \mathbb{R}, i=1, \ldots, p$, and $j=1, \ldots, k(\boldsymbol{\omega})$.

The proof is omitted due to space limitations.

With the aim to give an intuitive explanation of (11), note that the right hand side of the equation counts all possible partitions of the set $\mathcal{P}=\{1, \ldots, p\}$, while $C(s)$ in (13) accounts for the generic distribution of the variables $\tilde{\mathbf{x}}$. The quantity $w_{j}(\boldsymbol{\omega})$, instead, represents the indices pairing that appears in the exponent of the generic entry of the power $\mathbf{T}_{d}^{p}$.

\section{Convergence to the MarČEnKo-Pastur DISTRIBUTION}

In this section we show that the asymptotic eigenvalue distribution of the matrix $\mathbf{T}_{d}$ tends to the Marčenko-Pastur law [11], as $d \rightarrow \infty$. This is equivalent to prove that, as $d \rightarrow \infty$, the $p$-th moment of $\lambda$ tends to the $p$-th moment of the Marčenko-Pastur distribution, for every $p \geq 1$.

Theorem 5.1: Let $\mathbf{T}_{d}$ be a $(2 M+1)^{d} \times(2 M+1)^{d}$ Hermitian random matrix as defined in (9), where the properties of the random vectors $\mathbf{x}_{\mu(\mathbf{q})}$ are described in Section III. Let $\mathbb{E}\left[\lambda^{p}\right]$ be the $p$-th moment of the asymptotic eigenvalue distribution of $\mathbf{T}_{d}$, given by Theorem 4.1. Then, for any given $\beta$,

$$
\lim _{d \rightarrow \infty} \mathbb{E}\left[\lambda^{p}\right]=\mathbb{E}\left[\lambda_{\infty}^{p}\right]=\sum_{k=1}^{p} \beta^{p-k} N(p, k)
$$

where $N(p, k)$ are the Narayana numbers [20] and $\mathbb{E}\left[\lambda^{p}\right]$ are the Narayana polynomials, i.e., the moments of the MarčenkoPastur distribution [11].

The proof is omitted due to space limitations.
Example 3: We compute here the analytic expression of $\mathbb{E}\left[\lambda^{2}\right]$. Using (11), we get:

$$
\mathbb{E}\left[\lambda^{2}\right]=\sum_{k=1}^{2} \sum_{h=1}^{k} \beta^{2-h} \sum_{\boldsymbol{\omega} \in \Omega_{2, k}} \sum_{\boldsymbol{\omega}^{\prime} \in \Omega_{k, h}} u\left(\boldsymbol{\omega}^{\prime}\right) v\left(\boldsymbol{\omega}, \boldsymbol{\omega}^{\prime}\right)^{d}
$$

By expanding this expression and using the fact that $\Omega_{1,1}=\{[1]\}, \Omega_{2,1}=\{[1,1]\}, \Omega_{2,2}=\{[1,2]\}, u([1])=$ $1, u([1,1])=-1$, and $u([1,2])=1$ we obtain $\mathbb{E}\left[\lambda^{2}\right]$ as

$$
\beta v([1,1],[1])^{d}-\beta v([1,2],[1,1])^{d}+v([1,2],[1,2])^{d}
$$

We notice that, for $k=1, v([1,1],[1])=1$. The term $v([1,2],[1,2])$ refers instead to the case $k=h=2$, and it is given by

$$
v([1,2],[1,2])=\int_{\mathcal{H}_{2}} \prod_{j=1}^{2} \delta_{D}\left(w_{j}([1,2])\right) \mathrm{d} \mathbf{y}
$$

with $w_{1}([1,2])=y_{1}-y_{2}$ and $w_{2}([1,2])=y_{2}-y_{1}$. It follows that

$$
v([1,2],[1,2])=\int_{\mathcal{H}_{2}} \delta_{D}\left(y_{1}-y_{2}\right) \delta_{D}\left(y_{2}-y_{1}\right) \mathrm{d} \mathbf{y}=1
$$

Finally,

$$
\begin{aligned}
v([1,2],[1,1]) & =\int_{\mathcal{H}_{2}} \prod_{j=1}^{2} C\left(-\mathrm{j} 2 \pi \beta^{1 / d} w_{j}([1,2])\right) \mathrm{d} \mathbf{y} \\
& =\int_{\mathcal{H}_{2}}\left|C\left(-\mathrm{j} 2 \pi \beta^{1 / d}\left(y_{1}-y_{2}\right)\right)\right|^{2} \mathrm{~d} \mathbf{y}
\end{aligned}
$$

Thus, we write

$$
\mathbb{E}\left[\lambda^{2}\right]=1+\beta-\beta\left[\int_{\mathcal{H}_{2}}\left|C\left(-\mathrm{j} 2 \pi \beta^{1 / d}\left(y_{1}-y_{2}\right)\right)\right|^{2} \mathrm{~d} \mathbf{y}\right]^{d}
$$

\section{NUMERICAL RESULTS}

We can exploit our results on the asymptotic approximation to $f_{\lambda}$ to estimate the MSE on the reconstructed field. We assume that the sensor measurements are noisy and are given by $\mathbf{p}=\mathbf{s}+\mathbf{n}$ where $\mathbf{n}$ is a vector of noise samples with covariance matrix $\mathbb{E}\left[\mathbf{n n}^{\dagger}\right]=\sigma_{n}^{2} \mathbf{I}$. Also, let us denote by $\sigma_{a}^{2} \mathbf{I}$ the covariance matrix of the signal spectrum a. We define $\mathrm{SNR}_{m}=\sigma_{a}^{2} / \sigma_{n}^{2}$ as the signal-to-noise ratio on the sensor measurements. We consider the linear minimum mean square error (LMMSE) reconstruction technique, where the estimate of the spectrum a is obtained by filtering the noisy measures $p$ with the filter

$$
\left(\mathbf{G}_{d} \mathbf{G}_{d}^{\dagger}+\frac{1}{\mathrm{SNR}_{m}} \mathbf{I}\right)^{-1} \mathbf{G}_{d}
$$

Figure 1 shows the MSE obtained by using the LMMSE filter, as a function of $\mathrm{SNR}_{m}$. The curves labeled by " $d=1,2,3$ " refer to the case where the sampling points are quasi-equally spaced with jitter $\tilde{x}$, uniformly distributed over $[0,1)^{d}$, for $d=1,2,3$, and $\beta=0.729$. In this case, the results have been obtained by using the semi-analytical expression of the MSE 
given in [12], which is a function of $f_{\lambda}(d, \beta, z)$. The curve labeled by "Marčenko-Pastur" (thick line) reports the results derived through our asymptotic $(d \rightarrow \infty)$ approximation to the eigenvalue distribution, while the curve labeled by "Equally spaced" (dashed line) represents the MSE achieved under an equally spaced sensor placement, i.e., when the eigenvalue distribution is given by $f_{\lambda}(d, \beta, z)=\delta_{D}(z-1)$. Notice that the MSE grows as $d$ increases and tends to the MSE obtained by a Marčenko-Pastur eigenvalue distribution. Instead, as expected, the "Equally spaced" curve represents a lower bound to the system performance.

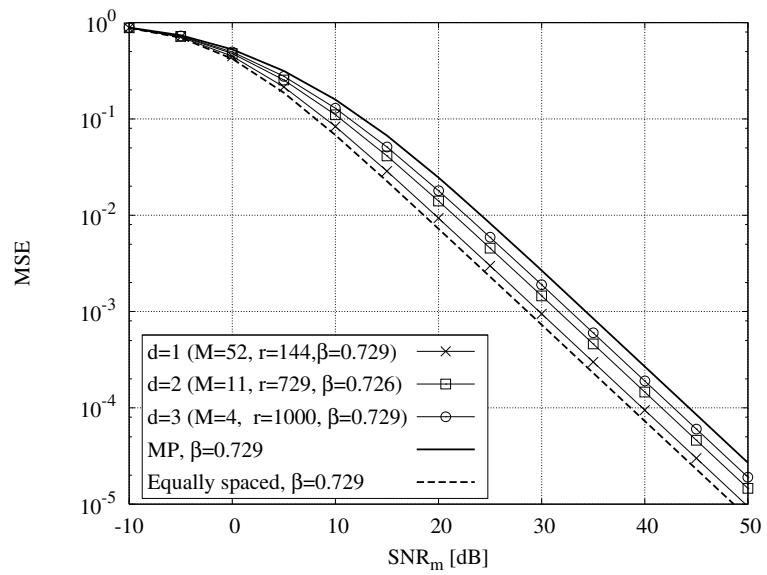

Fig. 1. MSE as a function of the signal-to-noise ratio on the sensor measurements for $d=1,2,3$. The curves are compared with the results obtained through our asymptotic analysis and with the equally spaced case

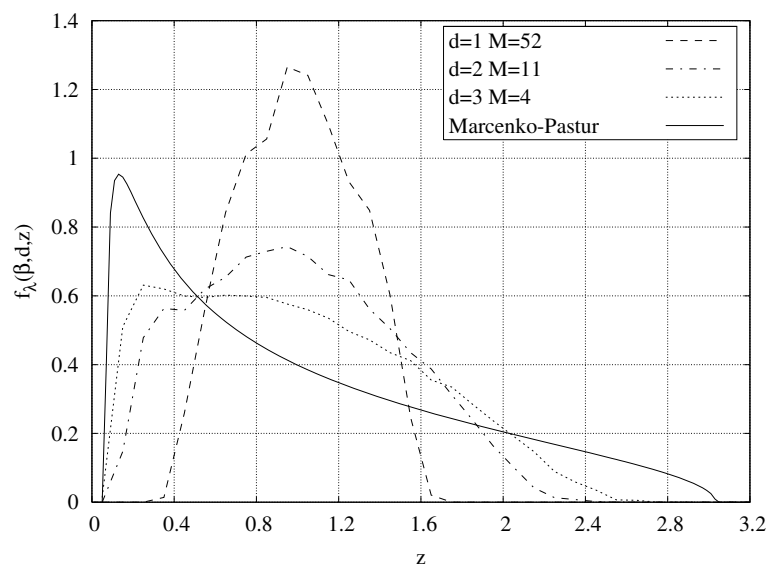

Fig. 2. Comparison between the Marčenko-Pastur distribution and the empirical distribution obtained for $\beta=0.55$ and $d=1,2,3$

Figure 2 shows the empirical eigenvalue distributions of the matrix $\mathbf{T}_{d}$ for $\beta=0.55, d=1,2,3$, and $\tilde{x}$ distributed in the hypercube $[0,1)^{d}$. The empirical distribution is compared to the Marčenko-Pastur distribution (solid line).

\section{CONCLUSIONS}

We studied the reconstruction of a bandlimited multidimensional signal from quasi-equally spaced measurements obtained from a wireless sensor network. As a measure of the reconstruction quality we used the mean square error. By using asymptotic analysis we derived the analytic expression of the moments of the eigenvalue distribution of a certain Toeplitz matrix characterizing the signal model. We also showed that, as the dimension of the signal approaches infinity, the eigenvalue distribution tends to the Marčenko-Pastur law. This result allowed to obtain a simple and accurate bound to the reconstruction MSE.

\section{ACKNOWLEDGMENT}

This work was supported partially by the European Commission in the framework of the FP7 Network of Excellence in Wireless COMmunications NEWCOM++ (contract n. 216715) and partially by the Regione Piemonte (Italy) through the VICSUM project.

\section{REFERENCES}

[1] A. Nordio, C.-F. Chiasserini, and E. Viterbo, "The impact of quasiequally spaced sensor layouts on field reconstruction," IPSN 2007, Cambridge, MA, Apr. 2007

[2] D. Ganesan, S. Ratnasamy, H. Wang, and D. Estrin, "Coping with irregular spatio-temporal sampling in sensor networks," ACM SIGCOMM, pp. 125-130, Jan. 2004.

[3] P. Zhao, C. Zhao, P. G. Casazza, "Perturbation of regular sampling in shift-invariant spaces for frames," IEEE Transactions on Information Theory, vol. 52, no. 10, pp. 4643-4648, Oct. 2006.

[4] D. S. Early and D. G. Long, "Image reconstruction and enhanced resolution imaging from irregular samples," IEEE Transactions on Geoscience and Remote Sensing, vol. 39, no 2, pp. 291-302, Feb. 2001.

[5] H. G. Feichtinger, K. Gröchenig, T. Strohmer, "Efficient numerical methods in non-uniform sampling theory," Numerische Mathematik, vol. 69, pp. 423-440, 1995.

[6] A. Nordio, C.-F. Chiasserini, and E. Viterbo "Quality of field reconstruction in sensor networks," INFOCOM, Anchorage, AK, May 2007.

[7] A. Tulino, S. Verdú, "Random matrix theory and wireless communications," Foundations and Trends in Communications and Information Theory, vol. 1, no. 1, 2004.

[8] M.L. Mehta, Random matrices, 2nd Edition, New York, Academic Press, 1991.

[9] K. Abed-Meraim, P. Loubaton, P. Moline's, "A subspace algorithm for certain blind identification problems," IEEE Trans. on Information Theory, vol. 43, pp. 499-511, Mar. 1997.

[10] Ø. Ryan and M. Debbah, "Free deconvolution for signal processing applications," http://arxiv.org/abs/cs.IT/0701025.

[11] V. A. Marčenko and L. A. Pastur, "Distribution of eigenvalues for some sets of random matrices," USSR Sbornik, Vol. 1, pp. 457-483, 1967.

[12] A. Nordio, C.-F. Chiasserini, and E. Viterbo, "Signal reconstruction in multidimensional sensor fields," IZS, Zurich, 2008.

[13] A. Nordio, A. Muscariello, and C.-F. Chiasserini, "Signal Compression and Reconstruction in Clustered Sensor Networks," ICC 2008, Beijing, China, 2008.

[14] Ø. Ryan and M. Debbah, "Random Vandermonde Matrices-Part I: Fundamental results", http://arxiv.org/abs/0802.3570v1

[15] Ø. Ryan and M. Debbah, "Random Vandermonde Matrices-Part II: Applications", http://arxiv.org/abs/0802.3572v1

[16] P. Billingsley, Probability and measure (3rd edition), John Wiley and Sons Inc, New York, 1995.

[17] L. Li, A. M. Tulino and S. Verdù, "Asymptotic eigenvalue moments for linear multiuser detection," Communications in Information and Systems, Vol. 1, No. 3, pp. 273-304, Sept. 2001.

[18] E.W. Weisstein, "Bell number," from MathWorld - A Wolfram Web Resource, http://mathworld.wolfram.com/BellNumber.html.

[19] E. W. Weisstein, "Stirling number of the second kind," from MathWorld - A Wolfram Web Resource, http://mathworld.wolfram.com/ StirlingNumberoftheSecondKind.html.

[20] I. Dumitriu and E. Rassart, "Path counting and random matrix theory," The Electronic Journal of Combinatorics, Vol. 10, No. 1, 2003. 